\title{
A DIGRAPH EQUATION FOR HOMOMORPHIE IMAGES
}

\author{
ROBERT D. GIRSE and RICHARD A. GILLMAN
}

Department of Mathematics

Idaho State University

Pocatello, Idaho 83209

(Received November 20,1985$)$

ABSTRACT. The definitions of a homomorphism and a contraction of a graph are generalized to digraphs. Solutions are given to the graph equation $\overline{\phi(D)}=\theta_{\phi}(\bar{D})$.

KEY WORDS AND PHRASES. Homomorphisms of graphs, contractions of graphs, digraphs. 1980 AMS SUBJECT CLASSIFICATION CODE. 05C20

By a graph G we mean a finite graph with no multiple edges or loops. If graphs $G$ and $\mathrm{H}$ are isomorphic we write $\mathrm{G}=\mathrm{H}$. An elementary homomorphism of a graph $\mathrm{G}$ is an identification of two non-adjacent vertices of $G$ and a homomorphism is a sequence of elementary homomorphisms. A homomorphism of $G$ onto $H$ preserves adjacency. Likewise, an elementary contraction of $G$ is the identification of two adjacent vertices of $G$ and a contraction is a sequence of elementary contractions [1]. Thus for every homomorphism $\phi$ of $G$ there is a related contraction $\theta_{\phi}$ of the complement of $G, \bar{G}$. This contraction is constructed as follows: $\phi$ is a sequence of elementary homomorphisms $\varepsilon_{1}, \varepsilon_{2}, \ldots, \varepsilon_{n}$ so we let $\theta_{\phi}$ be sequence of elementary contractions $\theta_{1}, \theta_{2}, \ldots, \theta_{n}$ where $\theta_{i}$ identifies the same vertices in $\bar{G}$ that $\varepsilon_{i}$ identifies in $G$.

Recently [2] the graph equation $\overline{\phi(G)}=\theta_{\phi}(\bar{G})$ was studied. In this paper, we generalize the definition of a homomorphism and its related contraction to digraphs and find general solutions to this graph equation. In doing so, we find an easier proof of the result given in [2].

A digraph $D$ consists of a finite vertex set $V(D)$ together with a set $E(D)$ of ordered pairs of distinct elements of $V(D)$, called arcs. Again, if $D_{1}$ is isomorphic to $D_{2}$ we write $D_{1}=D_{2}$. By an elementary homomorphism of $D$ we mean an identification of two mutually non-adjacent vertices of $D$ (neither uv nor vu are in $E(D)$ ). Similarly, an elementary contraction is an identification of two mutually adjacent vertices of $D$ (both uv and vu are in $E(D)$ ). A homomorphism (contraction) of $D$ is again a sequence of elementary homomorphisms (contractions). The contraction $\theta_{\phi}$ of $\bar{D}$ related to the homomorphism $\phi$ of $D$ is defined as for undirected graphs. 
We will use the following notation as need arises: $I b(u)$ is the set of vertices $v$ of $D$ such that $v u$ is an arc of $D, O b(u)$ is the set of vertices $v$ of $D$ such that uv is an arc of $D$, and $A(u)$ is the adjacency set of $u$ in the graph G.

THEOREM 1. Let $\varepsilon$ be an elementary homomorphism of $D$ identifying vertices $u_{1}$ and $u_{2}$. Then $\varepsilon(D)=\theta_{\varepsilon}(\bar{D})$ if and on $1 y$ if $I b\left(u_{1}\right)=I b\left(u_{2}\right)$ and $O b\left(u_{1}\right)=O b\left(u_{2}\right)$.

PROOF. Let $u=\varepsilon\left(u_{1}\right)=\theta_{\varepsilon}\left(u_{1}\right)$. First suppose that $O b\left(u_{1}\right) \neq O b\left(u_{2}\right)$. Excluding u as a possible endpoint of an arc, we have $v v^{\prime}$ is an arc of $\overline{\varepsilon(D)}$ if and only if $v v^{\prime}$ is an arc of $\theta_{\varepsilon}(\bar{D})$. Hence there is a one to one correspondence of those arcs in $\overline{\varepsilon(D)}$ without $u$ as an endpoint and those of $\theta_{\varepsilon}(\bar{D})$ without $u$ as an endpoint. The vertex $v$ of the arc uv must be in $o b\left(u_{1}\right) \cap o b\left(u_{2}\right),\left(o b\left(u_{1}\right) \cup o b\left(u_{2}\right)\right)^{c}$, or $O b\left(u_{1}\right) \nabla O b\left(u_{2}\right)$, the symmetric difference. In the first case, uv is not an arc of $\overline{\varepsilon(D)}$ or $\theta_{\varepsilon}(\bar{D})$ and in the second case, uv is an arc of both. The latter case implies that uv is not an arc of $\overline{E(D)}$ but is an arc of $\theta_{\varepsilon}(\bar{D})$. Thus for every vertex in $O b\left(u_{1}\right) \nabla O b\left(u_{2}\right), \theta_{\varepsilon}(\bar{D})$ has one more arc than $\overline{\varepsilon(D)}$. The same holds for vertices in $\operatorname{Ib}\left(u_{1}\right) \nabla \operatorname{Ib}\left(u_{2}\right)$. Thus if $O b\left(u_{1}\right) \neq O b\left(u_{2}\right)$ or $\operatorname{Ib}\left(u_{1}\right) \neq \operatorname{Ib}\left(u_{2}\right),\left|E\left(\theta_{\varepsilon}(\bar{D})\right)\right|>|E(\overline{\varepsilon(D)})|$ and hence $\overline{\varepsilon(D)} \neq \theta_{\varepsilon}(\bar{D})$. Now let $\operatorname{Ib}\left(u_{1}\right)=\operatorname{Ib}\left(u_{2}\right)$ and $O b\left(u_{1}\right)=O b\left(u_{2}\right)$. We will use the identity map from $V(\overline{\varepsilon(D)})$ onto $V\left(\theta_{\varepsilon}(\bar{D})\right)$ and hence need only consider arcs to and from $u$. If uv is in $E\left(\theta_{\varepsilon}(\bar{D})\right)$ then $u_{1} v$ and $u_{2} v$ are arcs in $\bar{D}$. Thus $u_{1} v$ and $u_{2} v$ are not arc of $D$ and subsequently uv is in $E(\overline{\varepsilon(D)})$. By the same argument, if uv is an arc of $\overline{\varepsilon(D)}$, uv will be an arc of $\theta_{\varepsilon}(\bar{D})$. This holds for arcs vu, so $\overline{\varepsilon(D)}=\theta_{\varepsilon}(\bar{D})$.

COROLLARY 1: $\overline{\phi(D)}=\theta_{\phi}(\bar{D})$ if and only if $\phi$ is a sequence of elementary homomorphisms, each of which satisfies the conditions of Theorem 1 .

A digraph $D$ is pseudo-complete $n$-partite if there is a partition $v_{1}, v_{2}, \ldots, v_{n}$ such that $u, u^{\prime}$ in $v_{i}$ for some $i$ implies $u$ and $u^{\prime}$ are mutually non-adjacent, if $u$ is an element of $v_{i}$ and $v$ is an element of $v_{j}$, $i \neq j$, then either uv or vu is an arc of $D$, and finally if $u$ and $u^{\prime}$ are in $v_{i}, v$ and $v^{\prime}$ are in $v_{j}, i \neq j$, and uv is an arc then uv', $u^{\prime} v$, and $u^{\prime} v^{\prime}$ are also.

THEOREM 2. $\overline{\phi(D)}=\theta_{\phi}(\bar{D})$ for all homomorphisms $\phi$ of $D$ if and only if $D$ is pseudo-complete n-partite.

PROOF. If $D$ is pseudo-completely n-partite, every elementary homomorphism identifies two vertices $u_{1}$ and $u_{2}$ in the same partition set and thus $\operatorname{Ib}\left(u_{1}\right)=\operatorname{Ib}\left(u_{2}\right)$ and $O b\left(u_{1}\right)=O b\left(u_{2}\right)$. Hence $\overline{\varepsilon(D)}=\theta_{\varepsilon}(\bar{D})$ for every elementary homomorphism and thus for every homomorphism of $D$. Conversely, partition $V(D)$ according to the relation: $u_{1}$ and $u_{2}$ are in $v_{i}$ if and only if $I b\left(u_{1}\right)=I b\left(u_{2}\right)$ and $O b\left(u_{1}\right)=O b\left(u_{2}\right)$. We need only show that if $u_{1}$ is in $v_{i}$ and $u_{2}$ is in $v_{j}, i \neq j$, then either $u_{1} u_{2}$ or $u_{2} u_{1}$ is in $E(D)$. Suppose $u_{1}$ and $u_{2}$ are mutually non-adjacent and let $\varepsilon$ be the elementary homomorphism identifying them. Since $\overline{\varepsilon(D)}=\theta_{\varepsilon}(\bar{D}), O b\left(u_{1}\right)=O b\left(u_{2}\right)$ and $\operatorname{Ib}\left(u_{1}\right)=\operatorname{Ib}\left(u_{2}\right)$ by Theorem 1 and hence $u_{1}$ and $u_{2}$ are in the same partition set. Thus if $u_{1}$ is in $v_{1}$ and $u_{2}$ is in $v_{j}, i \neq j$, there is an arc between them and $D$ must be pseudo-complete n-partite. 
If, for every vertex $u$ of $D, I b(u)=O b(u), D$ is a symmetric digraph and can be represented by a graph G. This leads to the following corollaries to Theorems 1 and 2 .

COROLLARY 2. An elementary homomorphism $\varepsilon$ identifying vertices $u$ and $v$ of $a$ graph G satisfies $\overline{\varepsilon(G)}=\theta_{\varepsilon}(\bar{G})$ if and only if $A\left(u_{1}\right)=A\left(u_{2}\right)$.

COROLlaRY 3. A homomorphism $\phi$ of $G$ satisfies $\overline{\phi(G)}=\theta_{\phi}(\bar{G})$ if and only if $\phi$ is a sequence of elementary homomorphisms, each satisfying Corollary 2.

COROLLARY 4. $\overline{\phi(G)}=\theta_{\phi}(\bar{G})$ for every homomorphism $\phi$ of $G$ if and only if $G$ is complete n-partite.

A study of the equation $\phi(D)=\theta_{\phi}(\bar{D})$ would be interesting, yet is apparently difficult considering the work done in [2] for graphs. We conjecture that if $D=\bar{D}$ and $\phi(D)=\theta_{\phi}(\bar{D}), \phi$ nontrivial, then $D$ is a symmetric digraph.

\section{REFERENCES}

1. HARARY, F. Graph Theory, Addison-Wesley, 1969.

2. GIRSE, R. D. Homomorphisms of Complete n-Partite Graphs, Internat. J. Math Math. Sci., 9 (1986), 193-196.

3. GIRSE, R. D. and GILLMAN, R. A. Homomorphisms and Related Contractions of Graphs, Internat. J. Math. Math. Sci., submitted. 


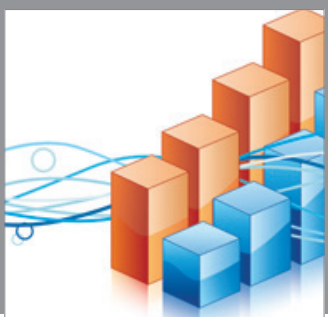

Advances in

Operations Research

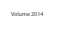

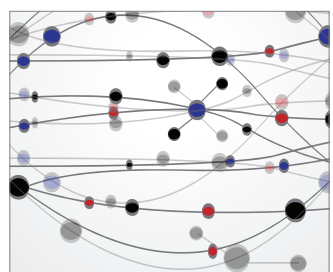

\section{The Scientific} World Journal
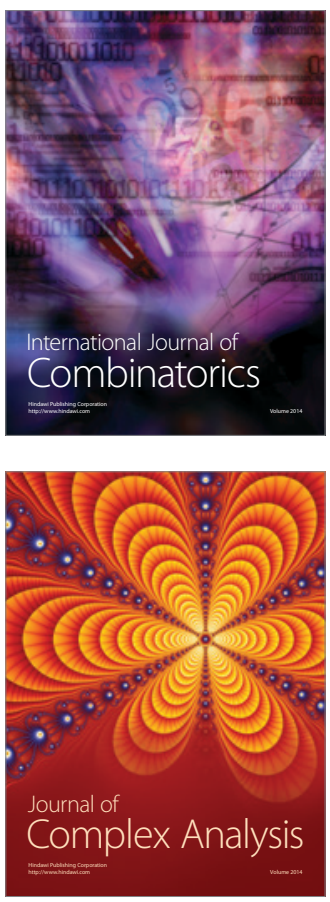

International Journal of

Mathematics and

Mathematical

Sciences
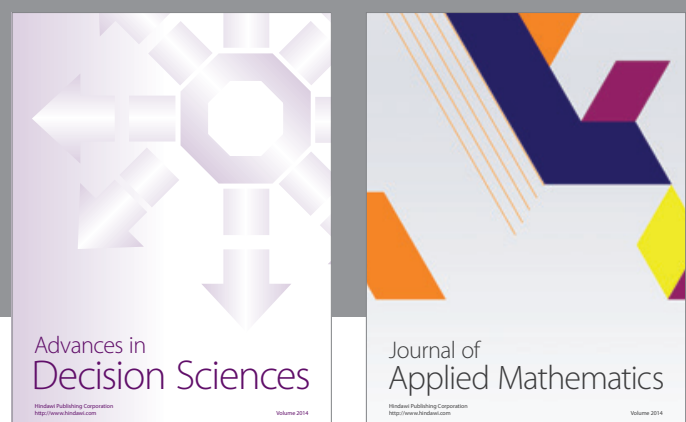

Journal of

Applied Mathematics
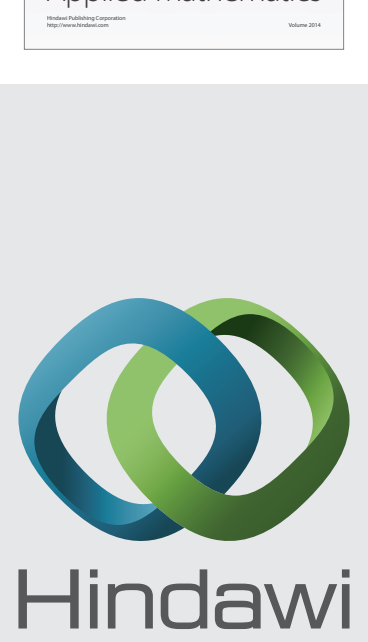

Submit your manuscripts at http://www.hindawi.com
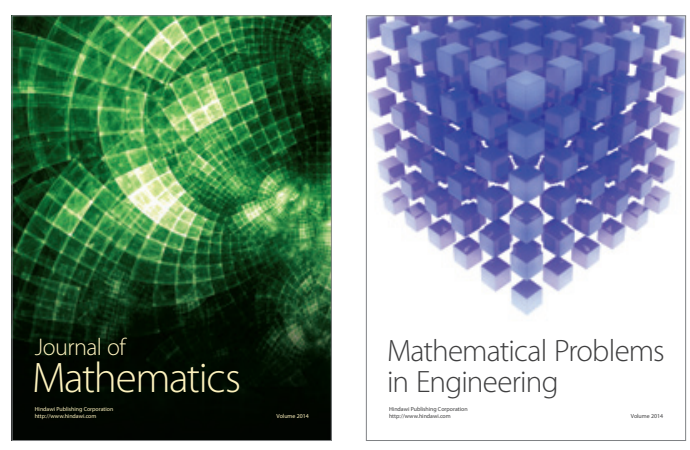

Mathematical Problems in Engineering
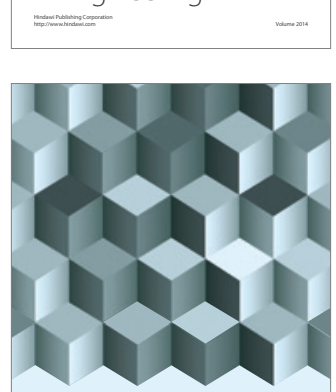

Journal of

Function Spaces
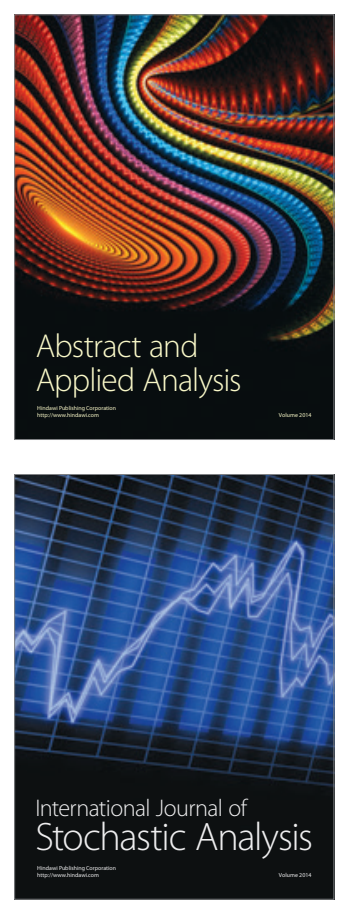

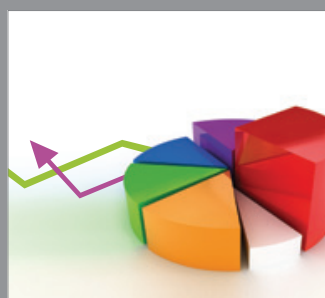

ournal of

Probability and Statistics

Promensencen
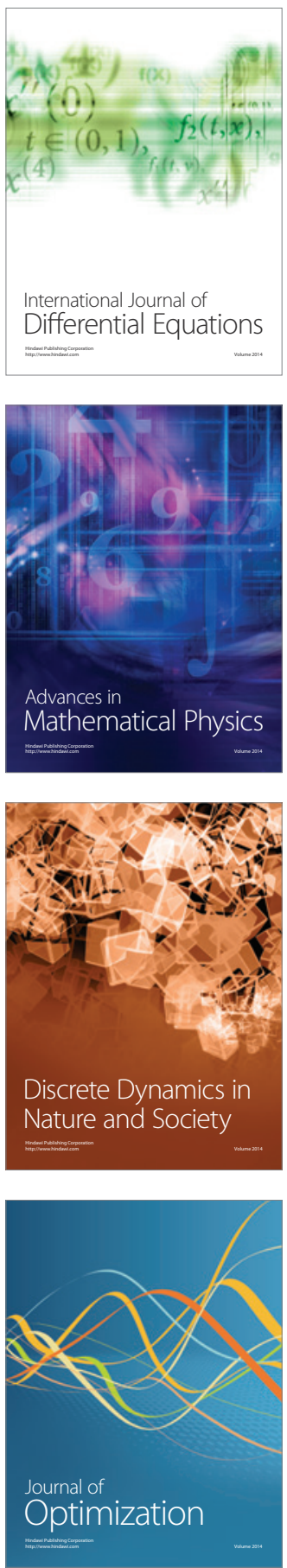